Article

\title{
Inactivation of the Pyrimidine Biosynthesis pyrD Gene Negatively Affects Biofilm Formation and Virulence Determinants in the Crohn's Disease-Associated Adherent Invasive Escherichia coli LF82 Strain
}

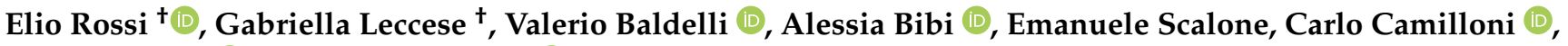 \\ Moira Paroni *(D) and Paolo Landini *(D) \\ Department of Biosciences, Università degli Studi di Milano, 20133 Milano, Italy; elio.rossi@unimi.it (E.R.); \\ gabriella.leccese@unimi.it (G.L.); valerio.baldelli@unimi.it (V.B.); alessia.bibi@unimi.it (A.B.); \\ emanuele.scalone@unimi.it (E.S.); carlo.camilloni@unimi.it (C.C.) \\ * Correspondence: moira.paroni@unimi.it (M.P.); paolo.landini@unimi.it (P.L.) \\ + These authors contributed equally to this work.
}

check for updates

Citation: Rossi, E.; Leccese, G.; Baldelli, V.; Bibi, A.; Scalone, E.; Camilloni, C.; Paroni, M.; Landini, P. Inactivation of the Pyrimidine Biosynthesis pyrD Gene Negatively Affects Biofilm Formation and Virulence Determinants in the Crohn's Disease-Associated Adherent Invasive Escherichia coli LF82 Strain. Microorganisms 2022, 10, 537. https://doi.org/10.3390/ microorganisms10030537

Academic Editor: Giovanni Di Bonaventura

Received: 24 January 2022

Accepted: 26 February 2022

Published: 28 February 2022

Publisher's Note: MDPI stays neutral with regard to jurisdictional claims in published maps and institutional affiliations.

Copyright: (C) 2022 by the authors. Licensee MDPI, Basel, Switzerland. This article is an open access article distributed under the terms and conditions of the Creative Commons Attribution (CC BY) license (https:// creativecommons.org/licenses/by/ $4.0 /)$.

\begin{abstract}
In Crohn's disease (CD) patients, the adherent-invasive Escherichia coli (AIEC) pathovar contributes to the chronic inflammation typical of the disease via its ability to invade gut epithelial cells and to survive in macrophages. We show that, in the AIEC strain LF82, inactivation of the pyrD gene, encoding dihydroorotate dehydrogenase (DHOD), an enzyme of the de novo pyrimidine biosynthetic pathway, completely abolished its ability of to grow in a macrophage environmentmimicking culture medium. In addition, pyrD inactivation reduced flagellar motility and strongly affected biofilm formation by downregulating transcription of both type 1 fimbriae and curli subunit genes. Thus, the pyrD gene appears to be essential for several cellular processes involved in AIEC virulence. Interestingly, vidofludimus (VF), a DHOD inhibitor, has been proposed as an effective drug in CD treatment. Despite displaying a potentially similar binding mode for both human and E. coli DHOD in computational molecular docking experiments, VF showed no activity on either growth or virulence-related processes in LF82. Altogether, our results suggest that the crucial role played by the pyrD gene in AIEC virulence, and the presence of structural differences between E. coli and human DHOD allowing for the design of specific inhibitors, make E. coli DHOD a promising target for therapeutical strategies aiming at counteracting chronic inflammation in CD by acting selectively on its bacterial triggers.
\end{abstract}

Keywords: adherent-invasive E. coli (AIEC); Crohn's disease; dihydroorotate dehydrogenase (DHOD); curli fibers; stress response; virulence; adhesion factors

\section{Introduction}

Crohn's disease $(\mathrm{CD})$ is characterized by chronic intestinal inflammation resulting from inappropriate and persistent activation of the intestinal mucosal immune system [1] The pathophysiology of $\mathrm{CD}$ is multifactorial, including genetic and environmental factors resulting in an aberrant immune response [2-6]. Arguably, however, one of the main factors in $\mathrm{CD}$ pathogenesis is gut microbiota dysbiosis $[7,8]$. Indeed, metagenomic analysis of human gut microbiota in $\mathrm{CD}$ patients has outlined clear pattern changes in microbial abundances in comparison to healthy individuals, such as a depletion of symbionts belonging to the Firmicutes phylum, like Bifidobacteria and Clostridia $[9,10]$. It is thought that these bacterial species might exert a protective effect by production of short chain fatty acids, such as butyrate, with anti-inflammatory effects [11]. In contrast, adherent-invasive Escherichia coli (AIEC) have consistently been found to be enriched in ileal specimens from $\mathrm{CD}$ patients in comparison to healthy subjects [12]. Although early reports suggested a more predominant role of AIEC in CD than in ulcerative colitis (UC), the other main 
inflammatory bowel disease, other works point to a role of the pathogen even in the latter disease $[13,14]$. The important role played by AIEC in IBD pathogenicity depends on their ability to invade intestinal epithelial cells and increase their permeability, triggering secretion of pro-inflammatory cytokines, thus ultimately resulting in chronic inflammation $[15,16]$. In addition, AIEC strains are able to survive and even replicate within human macrophages [17], leading to over-induction of the innate immune response. An extensive genetic and functional analysis, mostly carried out on the AIEC strain LF82, has identified several virulence factors and regulatory pathways involved in cell invasion and the induction of chronic inflammation in the human host, namely adhesion factors such as type 1 pili $[18,19]$, specific invasive genes such as ibeA [20], regulatory genes such as the $\sigma^{\mathrm{E}}$ network [21], and efflux pumps [22].

Due to the direct role played by microorganisms in the disease, antibiotic treatments are routinely used in Crohn's disease treatment: for instance, an association of metronidazole and of the anti-inflammatory drug azathioprine has been shown to be effective in preventive post-operative Crohn's recurrence [23]. Interestingly, azathioprine is itself an antimicrobial drug, able to inhibit growth of Mycobacterium avium ssp. paratuberculosis, another bacterium associated with Crohn's disease [24], and to impair virulence factors' production in AIEC [25]. However, the long-term effectiveness of antibiotic treatments is questionable, as they seem to further promote dysbiosis, thus not tackling, or sometimes even exacerbating, one of the main triggers for chronic inflammation [26]. To ameliorate dysbiosis, probiotics, especially bacteria of the Lactobacillus and Bifidobacterium genera, have been utilised on CD patients, but clinical studies on probiotic efficacy are inconclusive [27-29]. Recently, we have performed an in vitro study comparing the immunomodulatory effects of Lactobacilli and Bifidobacteria probiotic strains, showing that production of pro-inflammatory cytokines and the activation of the IL-23/Th17 axis in response to AIEC is effectively counteracted by probiotics in cells from healthy subjects and from individuals suffering with ulcerative colitis, but not in cells from CD patients [30], thus suggesting that probiotics might have a limited impact in CD.

In this manuscript, we report that a mutation inactivating the pyrD gene of the AIEC LF82 strain results in the loss of its ability to grow in a macrophage environment-mimicking medium and inhibits biofilm and virulence determinants, such as curli fibers, type 1 fimbriae, and flagellar motility. The pyrD gene encodes dihydroorotate dehydrogenase (DHOD), an enzyme involved in the de novo biosynthesis of pyrimidines. Thus, our results point to a role of intracellular pyrimidine concentrations as a regulatory signal for genes involved in virulence and in host interaction, as already identified for other pathogenic bacteria such as Pseudomonas aeruginosa and Shigella flexneri [31,32]. Based on the observation that $E$. coli DHOD not only differs from the human enzyme, but also has a low sequence identity to DHOD from probiotic species like Lactococcus lactis, we propose that the development of specific inhibitors of E. coli DHOD might be a potentially interesting therapeutic strategy for $\mathrm{CD}$ remission via selective inhibition of AIEC growth and virulence.

\section{Materials and Methods}

\subsection{Transposon Mutagenesis, Mutant Identification, and Screening on Acidic and Nutrient-Poor Medium}

Transposon insertion mutagenesis was carried out using the EZ-Tn5<R6K $\gamma$ ori/KAN-2> transposome (Lucigen, Middleton, WI, USA) on LF82, an AIEC strain originally isolated from an ileal biopsy specimen from a CD patient [33]. Transposon mutagenesis and the determination of a transposon insertion site by rescue cloning were carried out according to the manufacturer's instructions. Through multiple transformation rounds, we obtained a library of 10,058 kanamycin resistant mutants, i.e., a $2.2 \times$ coverage of the E. coli LF82 genome (4534 genes). Growth in acidic and nutrient-poor medium (Acid Medium: $100 \mathrm{mM}$ bis-Tris, $0.1 \%$ Casamino Acids, $0.16 \%$ glycerol, and $10 \mu \mathrm{M} \mathrm{MgCl}_{2}$, and the $\mathrm{pH}$ was adjusted to 5.8 with $10 \mathrm{M} \mathrm{HCl}$ [ [34] was determined as follows: overnight bacterial cultures grown 
in Yeast extract/Casamino acid (YESCA) medium (10 g/L casamino acids, $1.5 \mathrm{~g} / \mathrm{L}$ Yeast extract) at $37^{\circ} \mathrm{C}$ were diluted to $\mathrm{OD}_{600}=0.02$ in Acid Medium and overnight growth was constantly monitored in a microplate reader (SAFAS MP96).

\subsection{Adhesion Factor Detection, Biofilm Quantification, Motility Assay, MIC Determination and LPS Integrity Evaluation}

For phenotypical assays, bacteria were grown in YESCA medium, either at $30{ }^{\circ} \mathrm{C}$ or at $37^{\circ} \mathrm{C}$. When necessary, uracil was added at $0.25 \mathrm{mM}$ from a $50 \mathrm{mM}$ uracil solution in $50 \%$ dimethyl sulfoxide (DMSO); $0.25 \%$ DMSO was always added to control cultures. For adhesion factor detection, bacteria were grown on YESCA agar medium supplemented with either $0.004 \%$ Congo red and $0.002 \%$ Coomassie blue (CR) or $0.005 \%$ calcofluor (CF); dyes were always added to the medium after autoclaving. Bacteria were grown for $24 \mathrm{~h}$ at either $30^{\circ} \mathrm{C}$ or $37^{\circ} \mathrm{C}$; phenotypes were better detected after a further $24-48 \mathrm{~h}$ incubation at $4{ }^{\circ} \mathrm{C}$.

Biofilm formation was determined using the crystal violet $(\mathrm{CV})$ assay as described previously [25]. For flagellar motility assays, bacterial cells were grown overnight in YESCA and normalized to an $\mathrm{OD}_{600}=1$. For each culture, $3 \mu \mathrm{L}$ were spotted at the centre of a motility agar plate in the same growth medium used for overnight cultures, supplemented with $0.3 \%$ agar. Motility was determined by the diameter of the area colonized by the bacteria after $15 \mathrm{~h}$ of growth at $37^{\circ} \mathrm{C}$.

Determination of minimal inhibitory concentrations (MIC) of vidofludimus for LF82 was performed using standard 1:2 dilution methods in liquid YESCA medium, using an inoculum of $2 \times 10^{5} \mathrm{cfu} / \mathrm{mL}$. To assess vidofludimus antimicrobial activity on Gram positive bacteria, we performed MIC determination on Bacillus cereus strain 971 and Staphylococcus epidermidis ATCC 155.

To evaluate LPS integrity, overnight cultures of LF82 and LF82pyrD::Tn5 mutants grown in YESCA medium were normalized to $\mathrm{OD}_{600}=1.0$ and serially diluted 1:10 six times. $3 \mu \mathrm{L}$ of each dilution were spotted on LB and MacConkey agar in triplicates and plates were incubated overnight at $37^{\circ} \mathrm{C}$.

\subsection{Gene Expression Determination by Quantitative Real-Time PCR}

For RNA isolation, strains were grown either to mid-log phase or to the onset of the stationary phase in YESCA medium at $30^{\circ} \mathrm{C}$. Bacterial cells were harvested by centrifugation at $10,000 \times g$ for $5 \mathrm{~min}$ at $4{ }^{\circ} \mathrm{C}$ and cell pellets resuspended in $300 \mu \mathrm{L}$ of DNA/RNA Shield (Zymo Research, Irvine, CA, USA). Total RNA was extracted using the Quick-RNA Miniprep Kit (Zymo Research, Irvine, CA, USA) after the addition of $1 \mathrm{mg} / \mathrm{mL}$ Lysozyme and $400 \mu \mathrm{g} / \mathrm{mL}$ Proteinase $\mathrm{K}$ to the ruspended pellet. RNA samples were checked by agarose gel electrophoresis to assess lack of degradation and quantified spectrophotometrically. Genomic DNA removal and reverse transcription were performed on $1 \mu \mathrm{g}$ of total RNA, along with negative control samples incubated without reverse transcriptase using the QuantiTect Reverse Transcription Kit (Qiagen, Germantown, MD, USA). cDNA synthesis efficiency was verified by electrophoresis on agarose gel in comparison to negative controls. Real-time PCR was performed using the SYBR Green PCR master mixture, and the results were determined with a Rotor-Gene 3000 detection system (Corbett Research, Saffron Walden, UK). Reaction mixtures $(15 \mu \mathrm{L})$ included $0.1 \mu \mathrm{g}$ cDNA and $300 \mathrm{nM}$ primers in the reaction buffer and enzyme supplied by the manufacturer. Primer sequences are listed in Table S1. A minimum of three biologically independent experiments were considered for analysis; negative control samples (i.e., non-retrotranscribed RNA) never showed significant threshold cycles. The relative transcript amounts were determined using $16 \mathrm{~S}$ rRNA as the reference gene ([CtGene of interest-Ct16S] $=\Delta \mathrm{Ct}$ value) .

2.4. Computational Models for Vidofludimus Binding to Dihydroorotate Dehydrogenase (DHOD) from Different Organisms

Docking calculations were performed using Glide version 2021.3. Receptor structures (hDHOD, PDB code: 2PRL and EcDHOD, PDB code: 1F76) were prepared using the Protein 
Preparation Wizard of the Maestro graphical user interface (Schrödinger suite https: / www. schrodinger.com/, Schrödinger, Mannheim, Germany) by removing all crystallographic waters and additives and optimizing the orientation of hydrogen bonds and the protonation state of histidine, aspartic acid and glutamic acid. The protein preparation was followed by a restrained minimization of the whole system. Target grids were built on receptor structures. Ligand sampling was set to 'Flexible' and we included the Epik state penalties in the docking score. For each compound, 5 poses were saved after a post-minimization of the ligand structure within the binding site. The docking protocol was initially tested for its ability to reproduce the binding mode of the native R2C in the crystal structure (2PRL). The program was successful in reproducing the experimentally determined binding mode of the compound as it corresponds to the best-scored pose. Then, docking with VF was followed by a $50 \mathrm{~ns}$ long Molecular Dynamics (MD) simulation using the first predicted pose. MD simulations were performed using the Desmond package of the Schrödinger suite. The chosen force field was OPLS4. The systems were solvated in a dodecahedron hexagon box with a $10 \AA$ buffer and using TIP3P as water and $0.15 \mathrm{M}$ of $\mathrm{NaCl}$. MD simulation was preceded by energy minimization and equilibration steps.

\subsection{Statistical Analysis}

Statistical analysis was performed with Prism 9 software (GraphPad Software, San Diego, CA, USA). Student's $t$-tests for unpaired or paired samples were used to evaluate differences between means. Statistical significance between the means of more than two groups were performed using one-way ANOVA computing the Tukey's multiple comparisons test to evaluate differences between groups.

\section{Results}

\subsection{Mutant Selection in an Acidic and Nutrient Stress Medium Mimicking the Macrophage} Vacuole Environment

In order to identify genes that might be involved in AIEC virulence, we created a transposon mutagenesis library in the LF82 strain, using the EZ-Tn $5<\mathrm{R} 6 \mathrm{~K} \gamma$ ori $/ \mathrm{KAN}-2>$ transposome (Lucigen, WI, USA). Transposon insertion mutants were screened for their ability to grow in acid medium, recreating the harsh environment of the macrophage vacuole, characterized by low $\mathrm{pH}$ and limited nutrient availability [34]. Indeed, in this medium, even the LF82 parental strain was only able to carry out roughly two replications, with a maximal growth rate of $0.24 \mathrm{~h}^{-1}$ (Figure 1A and Figure S1). Out of the 10058 transposon mutants screened, 141 showed either a complete loss or a strong reduction in their ability to grow in acid medium, which was, however, often accompanied by growth defects also in the YESCA medium. Thus, we focused our attention on one mutant whose growth was not affected in YESCA, but totally abolished in acid medium. This mutant was compared to its parental LF82 strain in a more standardized experiment in which fresh overnight cultures of either strain grown in YESCA were resuspended to an $\mathrm{OD}_{600}=0.085$ and incubated overnight in acid medium, confirming total lack of growth by the mutant (Figure 1A).

The transposon insertion was mapped within the pyrD gene, encoding DHOD, the enzyme catalyzing the fourth step of the de novo pyrimidine biosynthesis, and the mutant strain will be referred to as LF82pyrD::Tn5 from now on. The transposon insertion site lies at nucleotide 368 of the 1011-bp long pyrD gene, immediately downstream of a portion of the gene coding for a domain involved in substrate binding, thus suggesting functional inactivation of the $p y r D$ gene (Figure 1B). Loss of $p y r D$ function was confirmed by restoration of LF82pyrD::Tn5 growth in acid medium by addition of $0.25 \mathrm{mM}$ uracil (Figure 1A), which would also suggest that absence of exogenous pyrimidines, rather than acid sensitivity, is the reason for LF82pyrD::Tn5 inability to grow in this medium. Indeed, AIEC mutants deficient in pyrimidine biosynthesis have already been shown to be unable to survive in macrophages [35]. Although exogenous uracil rescued its defective phenotype in acid medium, the LF82pyrD::Tn5 mutant still showed a longer lag phase (120 vs. $75 \mathrm{~min}$ ) 
and a slightly, albeit statistically significant, slower growth rate (0.21 vs. $\left.0.24 \mathrm{~h}^{-1}\right)$ than its parental strain even when exogenous uracil was provided (Figure 1A and Figure S1), suggesting that the pyrD mutation might affect LF82 fitness in acid medium even in the presence of excess pyrimidine availability.

A

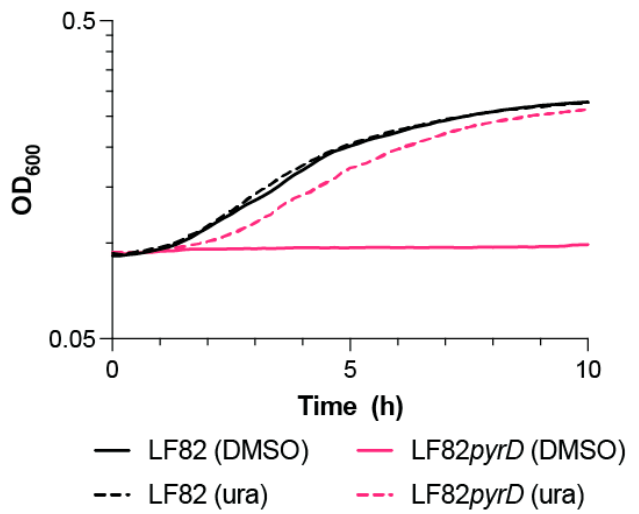

B

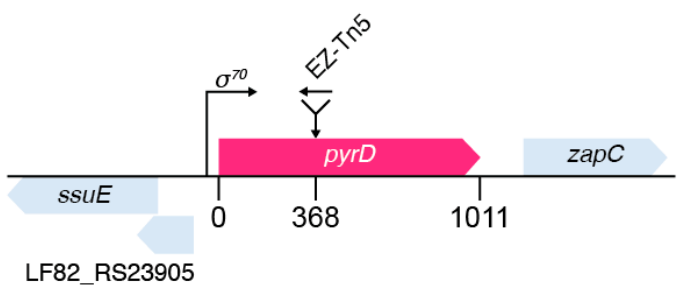

Figure 1. (A) Growth curves for LF82 (black) and LF82pyrD::Tn5 (LF82pyrD magenta) in acid medium mimicking the macrophage environment, either in the presence of $0.25 \mathrm{mM}$ uracil (dashed lines) or $0.25 \%$ dimethyl sulfoxide DMSO (solid lines); (B) Localization of the EZ-Tn5<R6K $\gamma$ ori/KAN-2> transposon insertion site in the pyrD gene.

\subsection{The LF82pyrD::Tn5 Mutant Is Impaired in Biofilm Formation and Adhesion Factors' Production and Displays a Slightly Reduced Flagellar Motility}

In previous works, we showed that perturbations of intracellular nucleotide pools strongly affect biofilm formation and adhesion factors' production in E. coli: indeed, in the $E$. coli MG1655 laboratory strain, mutations in de novo pyrimidine biosynthesis genes strongly downregulate curli production, a major adhesion factor [36]. Likewise, the purine analogue azathioprine, and other 6-mercaptopurine drugs, can impair both curli fibers' production and cell motility in LF82 [25].

To assess whether the pyrD mutation could also impair bacterial adhesion and cell motility, both involved in AIEC virulence, we performed crystal violet biofilm staining and swimming motility assays comparing LF82 to its LF82pyrD::Tn5 derivative (Figure 2A). Since curli fibers production in most E. coli strains, including LF82 [25], is strongly inhibited at $37^{\circ} \mathrm{C}$ in liquid media, we also performed biofilm and adhesion factor assays at $30^{\circ} \mathrm{C}$. Indeed, the LF82 wild type strain was more proficient in biofilm formation at $30{ }^{\circ} \mathrm{C}$ than at $37^{\circ} \mathrm{C}$, consistent with the production of curli fibers at lower growth temperatures (Figure 2A); however, the LF82pyrD::Tn5 mutant strain displayed a significant reduction in biofilm formation at both $30^{\circ} \mathrm{C}$ and $37^{\circ} \mathrm{C}$, possibly suggesting the inhibition of multiple adhesion factors by the pyrD mutation (Figure $2 \mathrm{~A}$ ). The impaired ability to form biofilm by the LF82pyrD::Tn5 mutant is not due to any reduction in overall growth in the YESCA medium (Figure S2).

To further confirm the effects of the LF82pyrD::Tn5 mutation on curli fibers production, we performed phenotypic assays on solid medium supplemented with either Congo red or Calcofluor, dyes that can bind both curli fibers and the extracellular polysaccharide cellulose, often co-produced with curli, as well as other cell surface structures. While LF82 showed, respectively, red and fluorescent phenotypes on either Congo red (CR)- or calcofluor (CF)-supplemented media, binding to either dye was affected in the LF82pyrD::Tn5 mutant, particularly at $37^{\circ} \mathrm{C}$ (Figure 2B), at which the mutant strain totally loses fluorescence on CF-supplemented medium and the pinkish coloration on CR displayed by its parental strain. Supplementation of $0.25 \mathrm{mM}$ uracil fully overcame the effects of the pyrD mutations, restoring the ability of LF82pyrD::Tn5 to bind either dye (Figure 2B), thus strongly suggesting that LF82pyrD::Tn5 phenotypes are indeed due to a reduction of the pyrimidine availability. 
A

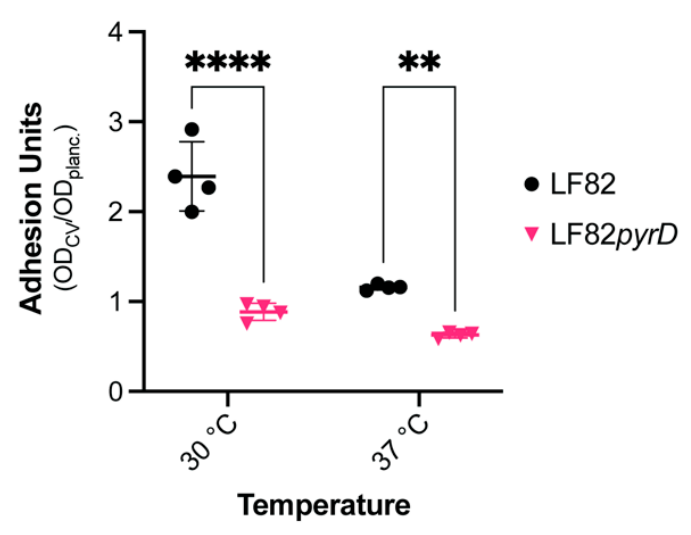

C

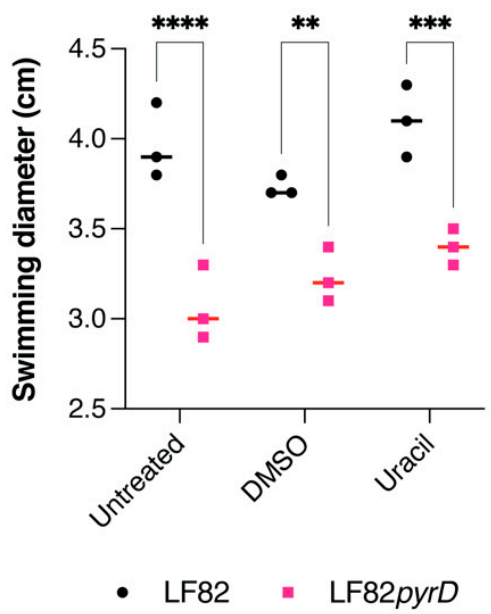

B

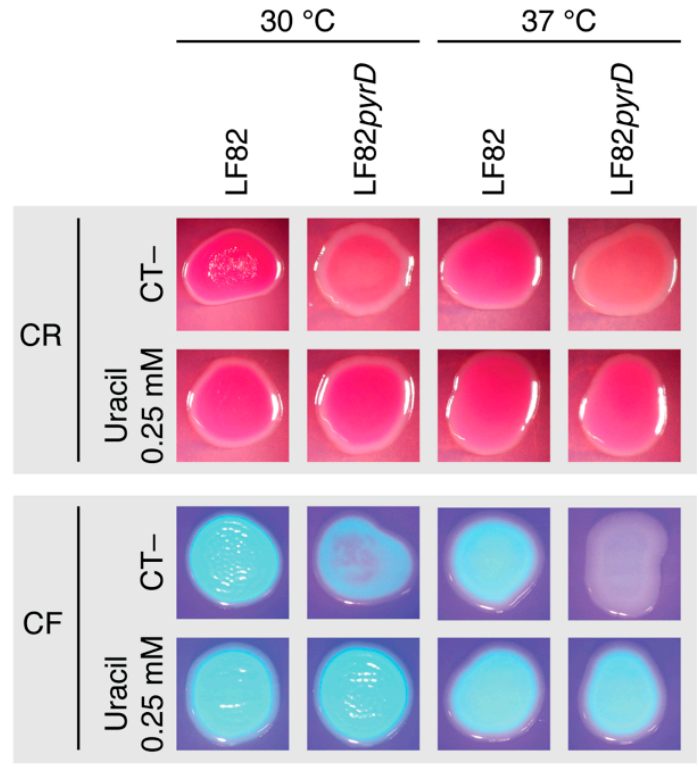

LF82
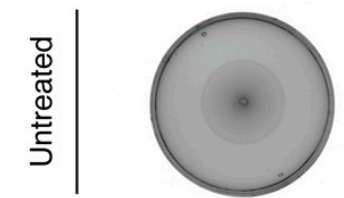

LF82pyrD


Figure 2. (A) Biofilm formation, measured as surface adhesion to polystyrene microtiter plates, using the Crystal violet method. Cultures were grown overnight in Yeast extract/Casamino acid (YESCA) medium at either $30{ }^{\circ} \mathrm{C}$ or $37{ }^{\circ} \mathrm{C}$. Results of four independent values and median are shown. ${ }^{* *}, p$-value $<0.01 ;{ }^{* * * *}, p$-value $<0.0001$, One-way ANOVA with Tukey's test for multiple comparisons; (B) LF82 and LF82pyrD::Tn5 (LF82pyrD) phenotypes grown either at $30^{\circ} \mathrm{C}$ or $37^{\circ} \mathrm{C}$ on YESCA medium, either with or without uracil supplementation, in the presence of either Congo red (CR) or Calcofluor (CF); (C) Swimming motility on YESCA soft agar plates (typical experiment, right) and measurement of halo diameters (three independent experiments and median are shown, left). $* *, p$-value $<0.01, * * *, p$-value $<0.001, * * * *, p$-value $<0.0001$, Two-way ANOVA with Šidák's test for multiple comparisons.

Finally, LF82pyrD::Tn5 is also impaired in cellular motility (Figure 2C), suggesting that perturbation of intracellular pyrimidine nucleotide pools also affects this important virulence-related cell process in AIEC [21]. Interestingly, however, unlike for CR and CF phenotypes (Figure 2B), uracil supplementation did not restore full cellular motility to the mutant strain, thus suggesting that the modulation of flagellar motility might depend on the relative concentrations of some de novo pyrimidine biosynthesis pathway intermediates rather than the overall pyrimidine intracellular concentration. 
Results in Figure 2 show that the LF82pyrD::Tn5 strain is hindered in production of adhesion factors, in biofilm formation and in cell motility. We reasoned that mutations in de novo pyrimidine biosynthesis might reduce the pool of UTP available for the activation of sugar precursors, thus affecting the production of extracellular polysaccharides such as peptidoglycan and lipopolysaccharide (LPS). In particular, mutations affecting LPS integrity have been shown to affect both adhesion to solid surfaces and cell motility in E. coli [37]. Thus, in order to verify whether the LPS structure might be impaired in the LF82pyrD::Tn5 strain, we determined its viability on MacConkey medium, as production of incomplete or aberrant LPS makes E. coli sensitive to bile salts present in this medium [38]. As shown in Figure S3A, we could not detect any reduction in viability comparing LF82pyrD::Tn5 strain growth in LB vs. MacConkey medium, thus suggesting that inactivation of the pyrD gene does not result in extensive perturbation of the LPS structure. As further assessment of the integrity of the cell envelope in the LF82pyrD::Tn5 mutant strain, we tested the activation of the $\sigma^{\mathrm{E}}$-dependent $l p t D$ gene [39], as the $\sigma^{\mathrm{E}}$ regulon is activated in response to changes in LPS structure [40]: again, no induction of $l p t D$ transcription was observed in the pyrD mutant strain (Figure S3B).

\subsection{The pyrD::Tn5 Mutation Results in Transcription Downregulation of Genes Encoding Curli Fibers and Type 1 Fimbriae}

Inhibition of curli production in the LF82pyrD::Tn5 mutant would be consistent with our previous observations that inactivation of genes of the de novo pyrimidine biosynthetic pathway negatively impacts curli and cellulose production in the E. coli MG1655 laboratory strain via the downregulation of the $\operatorname{csg} D E F G$ operon [36]. The first gene of the $\operatorname{csg} D E F G$ operon codes for the CsgD regulatory protein that activates transcription of the $\operatorname{csg} B A C$ operon, encoding curli structural subunits, and of the $a d r A(d g c C)$ gene, which in turn promotes cellulose production by acting on the product of the cellulose biosynthetic $b c s$ operon at the enzymatic level [41,42]. To verify this hypothesis, we compared expression of curli- and cellulose-related genes in LF82pyrD::Tn5 versus its parental strain. Gene expression was determined at $30^{\circ} \mathrm{C}$, both during exponential growth and at the onset of the stationary phase (Figure 3 ).

Inactivation of the $\operatorname{pyr} D$ gene strongly affected $\operatorname{csg} B$ expression, downregulating it by more than 10-fold, particularly at the onset of the stationary phase, in which $\operatorname{csg} B$ expression increase by 26 -fold compared to the exponential phase (Figure 3). In contrast, it did not significantly affect $\operatorname{csg} D$ transcription levels, which, in contrast, were higher during the exponential phase, showing a ca. 33-fold decrease at the onset of the stationary phase (Figure 3). Our results suggest that in AIEC, unlike the E. coli MG1655 laboratory strain, perturbation of intracellular pyrimidine pools due to $p y r D$ inactivation specifically targets $\operatorname{csg} B A C$, encoding curli subunits, without affecting the CsgD regulon at large. Indeed, transcription of the cellulose-related $a d r A$ and $b s c A$ genes were not significantly different in LF82pyrD::Tn5 compared to its parental strain (Figure 3, ca. 1.3 fold WT vs. mutant). However, it must be pointed out that $a d r A$ transcription levels were almost undetectable also in the LF82 parental strain, possibly suggesting that adrA might not be transcribed at significant levels in AIEC, at least in our experimental conditions.

In addition to the curli structural operon, the $p y r D:: \operatorname{Tn} 5$ mutation results in strong downregulation (ca. 20-fold in exponential phase, Figure 3) of the fim $A$ gene, encoding the major subunit of type 1 fimbriae, an important virulence factor in AIEC, promoting its adhesion to epithelial cells $[18,19]$, thus suggestted that perturbation of pyrimidine nucleotide pools negatively affects multiple adhesion factors in LF82. In contrast, fliC, encoding the main flagellar subunit, was not differently expressed in the LF82pyrD::Tn5 mutant, suggesting that reduced motility in this strain (Figure 2C) might be mediated at flagellar motility rather than at the flagellar gene transcription level. 
$\operatorname{csg} B$

$\operatorname{csg} D$

fimA
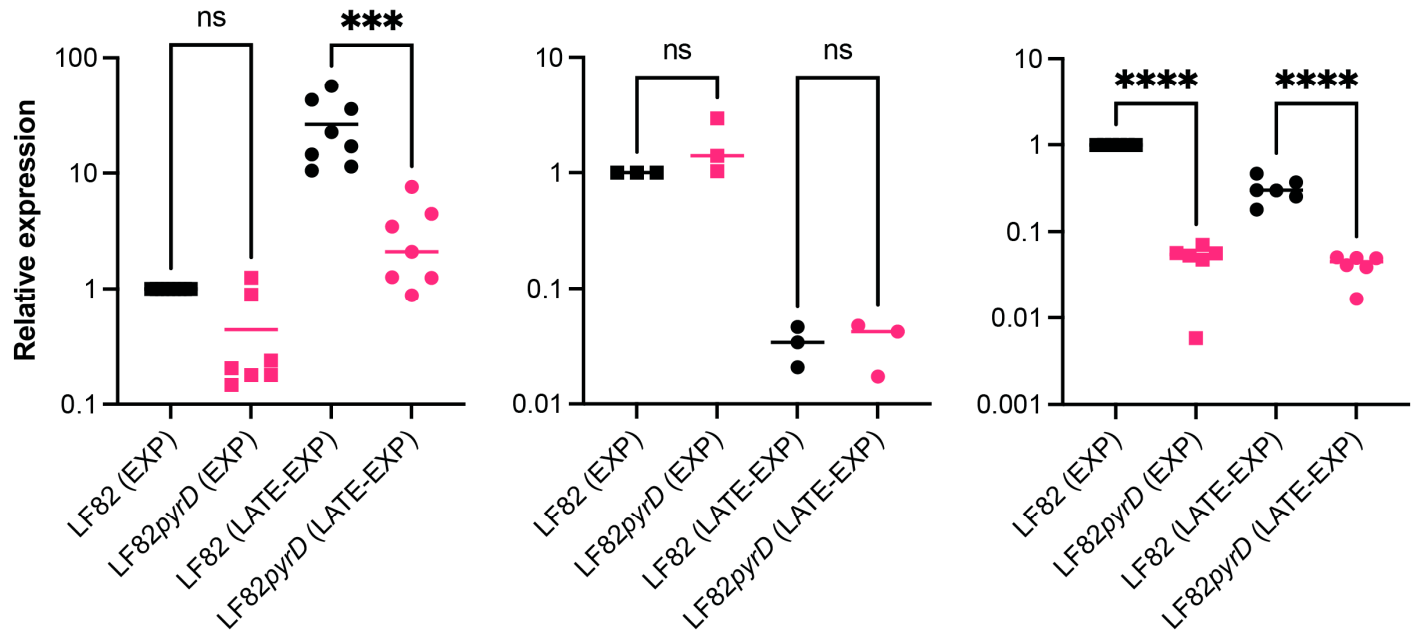

flic

bcsA
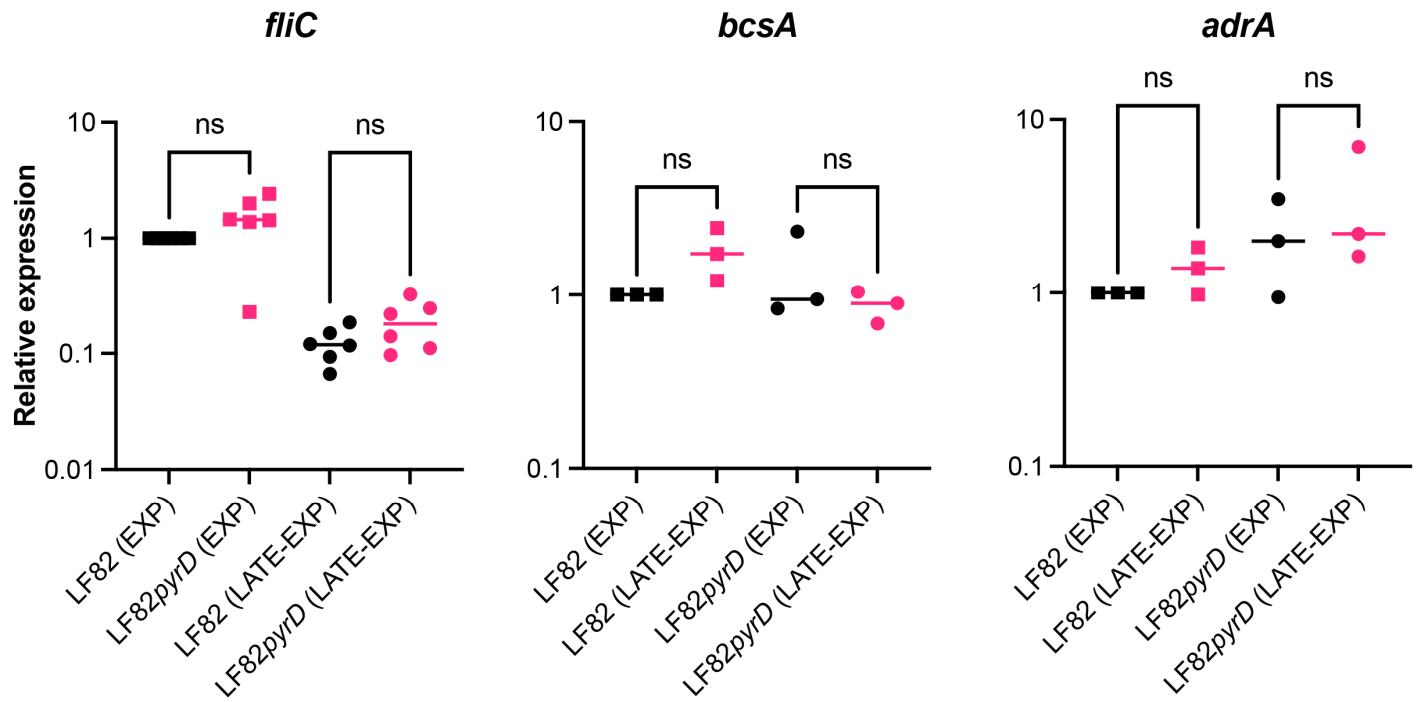

Figure 3. Determination of gene expression levels in the LF82 vs. the LF82pyrD::Tn5 (LF82pyrD) strain by qRT-PCR. RNA was extracted from bacterial cultures grown in YESCA medium at $30{ }^{\circ} \mathrm{C}$ either during exponential growth $\left(\mathrm{EXP}, \mathrm{OD}_{600 \mathrm{~nm}}=0.4\right)$ or transition to stationary phase (LATE-EXP, $\mathrm{OD}_{600 \mathrm{~nm}}=1.0$ ). Values are expressed as arbitrary units; transcription levels in the LF82 strain during exponential growth are set to 1 . Data are from at least three independent experiments and median are shown. ns, not significant; ${ }^{* *}, p$-value $<0.001,{ }^{* * * *}, p$-value $<0.0001$ One-way ANOVA with Tukey's test for multiple comparisons.

\subsection{Dihydroorotate Dehydrogenase (DHOD) as Potential Drug Target}

Results presented so far suggest that inactivation of the $\operatorname{pyrD}$ gene, resulting in loss of dihydroorotate dehydrogenase (DHOD) activity, and consequent perturbation of intracellular pyrimidine pools, affects a variety of cellular processes involved in AIEC host colonization and virulence, such as cell adhesion and motility (Figure 2), and ability to grow in environments devoid of exogenous pyrimidines (Figure 1), such as the vacuoles formed during macrophage infection by AIEC [35]. Interestingly, inhibitors of the human DHOD protein, like vidofludimus (VF), have been widely studied as anti-inflammatory drugs in several pathologies, including CD [43]. We recently showed that purine synthesis inhibitors 6-mercaptopurines, a class of widely used anti-inflammatory drugs, possess antimicrobial activity against LF82 and can inhibit biofilm formation and motility in this bacterium at subinhibitory concentrations for growth [25], thus suggesting that their antimicrobial activity might contribute to their effectiveness in Crohn's disease treatment. We 
hypothesized that VF might also inhibit $E$. coli DHOD, thus mimicking the effects of the pyrD mutation and hampering AIEC virulence, and that such inhibition might contribute to its anti-inflammatory action.

Unlike 6-mercaptopurines, however, VF showed no antibacterial activity on LF82 up to $256 \mu \mathrm{g} / \mathrm{mL}$ (Figure 4B), and it failed to inhibit biofilm formation at either $30{ }^{\circ} \mathrm{C}$ or $37^{\circ} \mathrm{C}$ (Figure $4 \mathrm{C}$ ), while only promoting a very slight phenotypic change of LF82 on CR-supplemented media at $37^{\circ} \mathrm{C}$ (Figure 4A). VF even induced a slight increase in biofilm formation that was only statistically significant at $37^{\circ} \mathrm{C}$ and did not show a clear dose-dependence, suggesting that these effects are not mediated by inhibition of DHOD activity. The lack of significant biological effects on LF82 by VF might be due either to inability to enter the bacterial cells of Gram negative bacteria, despite its small molecular size (molecular mass $=355.12$ ), or to low binding affinity to bacterial DHOD. VF showed very poor antimicrobial activity on the Gram positive bacteria Bacillus cereus and Staphylococcus epidermidis (MIC $=128 \mu \mathrm{g} / \mathrm{mL}$ for either species, Figure 4B), which are typically more sensitive to antimicrobial agents not able to cross the outer membrane of Gram negative bacteria, possibly suggesting poor inhibition of bacterial DHOD. Even in M9Glucose minimal medium, in which pyrimidine nucleotides are exclusively synthesized by the de novo biosynthetic pathway, and thus growth is totally dependent on the DHOD activity, VF showed no antimicrobial activity against E. coli LF82 (Figure 4B).

A

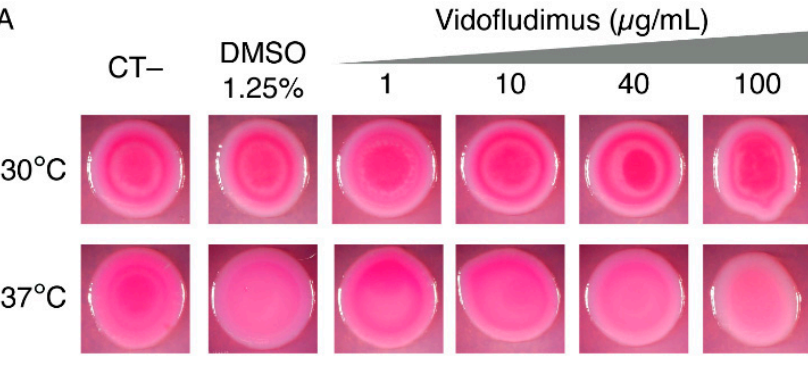

B

\begin{tabular}{|l|c|}
\hline Species & MIC $(\mu \mathrm{g} / \mathrm{mL})$ \\
\hline Staphylococcus epidermidis & 128 \\
\hline Bacillus cereus & 128 \\
\hline Escherichia coli MG1655 & $>256$ \\
\hline Escherichia coli LF82 & $>256$ \\
\hline Escherichia coli LF82 (M9 Glu) & $>256$ \\
\hline
\end{tabular}

$30{ }^{\circ} \mathrm{C}$

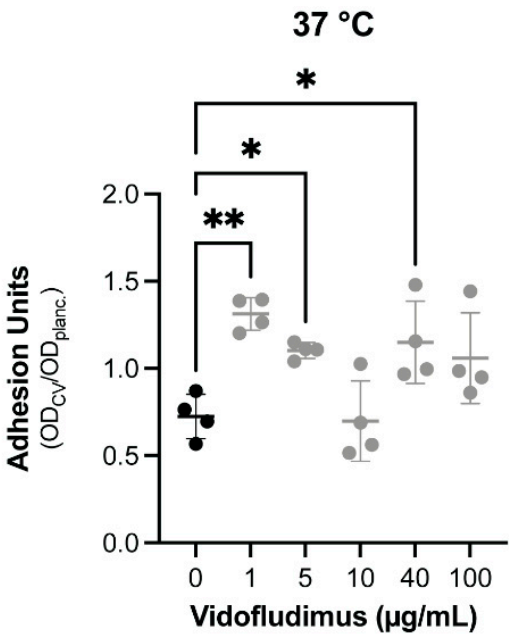

Figure 4. Effects of vidofludimus (VF) on LF82 phenotypes and on microbial growth. (A) Phenotypes on Congo red-supplemented YESCA agar medium, either at $30^{\circ} \mathrm{C}$ or $37^{\circ} \mathrm{C}$, in the presence of growing VF concentrations. The final DMSO concentration was kept at $1.25 \%$ for all VF concentrations tested. (B) Minimal inhibitory concentrations (MIC) on E. coli (LF82, in YESCA and M9 Glu 0.2\% media, and the laboratory strain MG1655, in YESCA medium) and the Gram positive bacteria Bacillus cereus and Staphylococcus epidermidis (in YESCA medium). (C) Biofilm formation, measured as surface adhesion to polystyrene microtiter plates, using the Crystal violet method, in the presence of increasing VF concentrations. Cultures were grown overnight in YESCA medium at either $30{ }^{\circ} \mathrm{C}$ or $37^{\circ} \mathrm{C}$. ${ }^{*}, p$-value $<0.05,{ }^{* *}, p$-value $<0.01$ One-way ANOVA with Tukey's test for multiple comparisons. Only significant values are shown. 


\subsection{In Silico Analysis of Vidofludimus/DHOD Interaction}

To further evaluate VF-DHOD interactions, we carried out in silico molecular docking experiments using GLIDE (see Materials and Methods). First we redocked the ligand 5-methoxy-2-[(4-phenoxyphenyl)amino]benzoic acid (R2C) to the corresponding experimentallydetermined structure (PDB ID: 2PRL). The ligand shows a GLIDE score of -9.6, with a binding pose identical to the experimental one, where the main interaction is between the carboxylic acid of R2C and R136 and Q47 along with many other polar and non-polar interactions. Then, we docked VF to both human and E. coli DHOD (hDHOD and EcDHOD, respectively). Docking on hDHOD showed a GLIDE score of -9.4 with a binding pose comparable to R2C. In particular, the interaction between VF carboxylic acid and R136 and Q47 is maintained (Figure 5A).
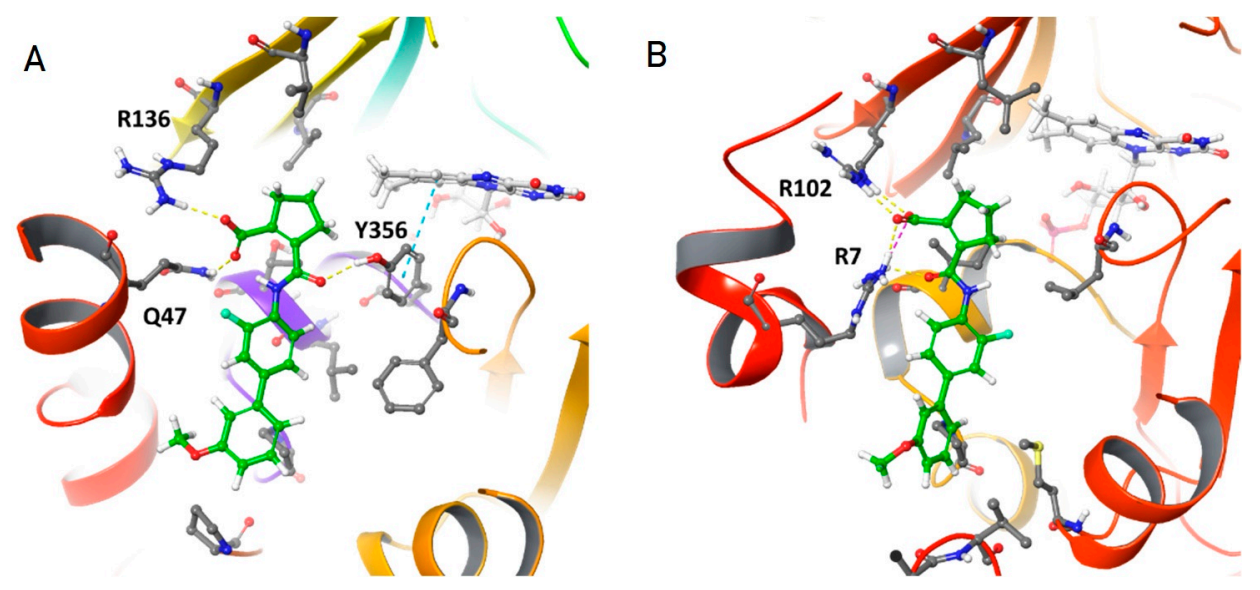

Figure 5. Model of VF-DHOD interaction. (A) VF (in green) docked to hDHOD showed an interaction between the VF carboxylic acid moiety and both R136 and Q47. A third interaction is observed between the amide group of VF and the hydroxyl group of Y356. (B) When VF is docked to EcDHOD we observed that the VF carboxylic acid moiety form interactions with R102 (equivalent to R136 in human) and R7 (in place of Q47 in human). The VF amide group does not form any interaction.

Furthermore, a third interaction is formed between the same carboxylic moiety with the hydroxyl group of Y356. Docking to EcDHOD again showed a comparable binding pose (Figure 5B). The carboxylic moiety of VF can interact with a conserved R102 (R136 in human) and with R7 (P44 in human) while lacking a third interaction present in the former. In addition, polar and non-polar interactions are also less optimised, resulting in a reduced GLIDE score of -8 . Of note, EcDHOD and hDHOD are structurally similar with an RMSD of less than $1 \AA$ and a sequence identity of $41 \%$. Based on these results, we expect VF to be able to interact with both $\mathrm{hDHOD}$ and EcDHOD with similar binding affinities, which would be consistent with the hypothesis that the lack of VF antimicrobial activity might be due to its inability to enter bacterial cells. In contrast, a structural comparison between $\mathrm{hDHOD}, \mathrm{EcDHOD}$ and the structures of the two DHOD isoenzymes in the Gram positive bacterium Lactococcus lactis (the pyrD homodimer and the pyrD-pyrK heterotetramer, 2DOR and 1EP2, respectively) show that in either DHOD form from this bacterium, the predicted binding site for VF is missing due to the lack of the two N-terminal helices necessary for DHOD-VF interaction (Figure S4).

\section{Discussion}

The ability of intracellular pathogenic bacteria such as AIEC to invade the gut epithelium and to survive in macrophages is a major trigger for chronic gut inflammation in CD. Thus, specific inhibition of AIEC virulence could be a promising strategy in CD treatment, as it might counteract inflammation without exacerbating gut dysbiosis, which can instead be observed in response to antibiotic therapy [26]. 
In order to identify potential targets for anti-virulence agents, we have generated a transposon insertion library in the AIEC strain LF82 and looked for mutants unable to survive in a medium mimicking the macrophage environment. Through this approach, we selected an insertion mutation in the $p y r D$ gene (LF82pyrD::Tn5 mutant), encoding for DHOD, an enzyme part of the de novo pyrimidine biosynthetic pathway. The selection of the pyrD mutant is likely due to the lack of exogenous pyrimidines in the medium used in our screening (Figure 1); however, it confirms previous results showing that mutants in AIEC, as well as in Salmonella, impaired in their de novo pyrimidine biosynthetic pathway, are indeed unable to survive in macrophages $[35,44]$, which is in line with the more general notion that, in several intracellular pathogens, auxotrophic mutants, either for nucleotides or for some amino acids, are avirulent, mostly because of their inability to grow or survive [45-47].

The pleiotropic effects of mutations in the pyrimidine biosynthetic pathway, such as the inability to form biofilm or to produce virulence factors, have already been described in the MG1655 laboratory strain of E. coli [36] as well as in other bacteria, such as P. aeruginosa [32], possibly via sensing of intracellular pyrimidine nucleotide pools by global regulators [48]. In this work, we showed that, in LF82, inactivation of the pyrD gene resulted in the inhibition of surface attachment (Figure 2) via downregulation of genes encoding curli fibers (Figures 2 and 3) and type 1 fimbriae (Figure 3) which are, arguably, the two main proteinaceous adhesion factors in E. coli. We had already described that, in E coli MG1655, mutations in the pyrimidine biosynthetic genes strongly affected transcription of the csgDEFG operon [36], which includes the CsgD regulatory protein presiding to curli and cellulose production; in contrast, in the LF82 background, gene expression downregulation by pyrD inactivation was only observed for the $\operatorname{csg} B A C$ operon, encoding curli structural subunits. This observation would suggest that, in LF82, sensing the lack of intracellular pyrimidines might be more selectively relayed towards turning off curli production, rather than to a more general effect on the CsgD regulon (Figure 3). Inhibition of curli production might be clinically relevant as, although curli production is turned off at $37^{\circ} \mathrm{C}$ in laboratory conditions, antibodies against these structures have been isolated in the context of various diseases, suggesting their production would indeed take place during infection. Indeed, curli binding to the TLR1/2 Toll-like receptors and activation of the NOD-like receptor protein 3 inflammasome are thought to contribute to overall inflammation in CD [49,50].

While the role of curli fibers in AIEC infection has not yet been fully characterised, type 1 fimbriae are considered bona fide AIEC virulence factors that are able to promote bacterial adhesion to epithelial cells [18]. Interestingly, the expression of both curli fibersand type 1 pili-encoding genes are negatively regulated in the presence of glucose in LF82, suggesting positive control by the CAP master regulator [25], whose activity appears to respond also to pyrimidine intracellular concentrations, as recently proposed [51]. At any rate, the inhibition of pyrimidine biosynthesis appears to have a very extensive impact on AIEC adhesion factors, reiterating its potential as a target for antimicrobial drugs.

Finally, in addition to survival in macrophages, biofilm formation and curli production, as well as cellular motility, another virulence factor in AIEC, was also affected by pyrD inactivation, probably via regulation of flagellar motility, as transcription of the fliC gene, part of the main flagellar operon, was unaffected in the LF82pyrD::Tn5 mutant strain (Figure 3). Unlike survival in the macrophage-mimicking medium and adhesion factors' production, flagellar motility was not fully restored by uracil supplementation (Figure 2C), possibly suggesting that accumulation of early intermediates of the de novo pyrimidine biosynthesis, rather than pyrimidine availability, might be involved in this process. Indeed, pyrimidine biosynthesis intermediates such as N-carbamoyl-aspartate can modulate production of c-di-GMP, a signal molecule involved in several processes, including flagellar motility [52].

The pleiotropic effects of the pyrD mutation on several virulence-related processes would make DHOD, the product of the pyrD gene, a suitable target for novel inhibitors of AIEC growth and/or virulence. This led us to hypothesize that VF, a known inhibitor of 
human DHOD studied in clinical trials as an anti-inflammatory drug in the treatment of $\mathrm{CD}[43,53]$, could possess antimicrobial and antivirulence activity against AIEC via bacterial DHOD inhibition. Indeed, antimicrobial activity against microorganisms able to trigger chronic inflammation in $\mathrm{CD}$ has been proposed to be at least partly responsible for the antiinflammatory activity of mercaptopurines, which, like VF, can inhibit de novo nucleotide biosynthesis in bacteria $[24,25,54]$. However, unlike mercaptopurines, VF possesses little or no activity either against AIEC or Gram positive microorganisms (Figure 4), although molecular docking analysis (Figure 5) predicts that VF can bind human and E. coli DHOD with similar affinity. The lack of antimicrobial activity by VF against AIEC (Figure 4) might thus depend on VF poor penetration into the cell, despite its low molecular mass (355.12), a behaviour observed also for several antimicrobial agents, such as clindamycin (MW 424.98) [55]. In contrast, comparison of the E. coli DHOD structure to both DHOD isoenzymes of the Gram positive, probiotic bacterium Lactococcus lactis (Figure S4) shows the absence of any potential binding site for VF in either DHOD isoform. Our results reiterate previous observations on the functional diversity of DHOD among bacteria [56] which, in addition to known structural differences between human and E. coli DHOD [57], would suggest the possibility to design DHOD inhibitors that might specifically target only a subset of selected bacterial species. In the context of a chronic inflammatory disease such as $\mathrm{CD}$, targeting E. coli DHOD without affecting beneficial bacteria in the gut microbiota could eradicate an important trigger for chronic inflammation and help restore gut microbiota eubiosis, thus representing a promising therapeutic strategy.

Supplementary Materials: The following are available online at http:/ /www.mdpi.com/article/10.3390/ microorganisms10030537/s1, Table S1: List of oligonucleotides used for qRT-PCR experiments. Figure S1: Growth rates and lag phases of LF82 wild type strain and of the LF82pyrD::Tn5 mutant in Acid medium supplemented either with DMSO or uracil. Figure S2: Optical density of overnight cultures of LF82 wild type strain and of the LF82pyrD::Tn5 mutant in YESCA medium in microtiter plates used for biofilm determination experiments. Figure S3: (A) Viability of the LF82pyrD::Tn5 mutant compared to its parental strain on LB Agar and MacConkey media. (B) Relative expression levels of the lptD gene. Figure S4: structures of Lactococcus lactis PyrD protein.

Author Contributions: Conceptualization, E.R., P.L., M.P.; Funding acquisition, M.P.; Investigation, E.R., G.L., V.B., A.B., E.S., C.C.; Resources, M.P., P.L.; Supervision, E.R., M.P., P.L.; Writing-original draft, P.L.; Writing-review and editing, E.R., M.P., P.L. All authors have read and agreed to the published version of the manuscript.

Funding: This work was funded by Cariplo Foundation grant n. \#2017-0816 to MP and by a Fondazione Telethon grant (GP191334) to CC.

Institutional Review Board Statement: Not applicable.

Informed Consent Statement: Not applicable.

Data Availability Statement: All data generated or analyzed during this study are included in this published article (and its supplementary information files).

Acknowledgments: We would like to thank Sara Biella for her assistance in carrying out the experimental work.

Conflicts of Interest: The authors declare that they have no conflict of interest.

\section{References}

1. de Souza, H.S.P.; Fiocchi, C. Immunopathogenesis of IBD: Current State of the Art. Nat. Rev. Gastroenterol. 2016, 13, 13-27. [CrossRef] [PubMed]

2. Duerr, R.H.; Taylor, K.D.; Brant, S.R.; Rioux, J.D.; Silverberg, M.S.; Daly, M.J.; Steinhart, A.H.; Abraham, C.; Regueiro, M.; Griffiths, A.; et al. A Genome-Wide Association Study Identifies IL23R as an Inflammatory Bowel Disease Gene. Science 2006, 314, 1461-1463. [CrossRef] [PubMed]

3. Villani, A.-C.; Lemire, M.; Fortin, G.; Louis, E.; Silverberg, M.S.; Collette, C.; Baba, N.; Libioulle, C.; Belaiche, J.; Bitton, A.; et al. Common Variants in the NLRP3 Region Contribute to Crohn's Disease Susceptibility. Nat. Genet. 2009, 41, 71-76. [CrossRef] [PubMed] 
4. $\quad$ Ng, S.C.; Bernstein, C.N.; Vatn, M.H.; Lakatos, P.L.; Loftus, E.V.; Tysk, C.; O'Morain, C.; Moum, B.; Colombel, J.-F.; on behalf of the Epidemiology and Natural History Task Force of the International Organization of Inflammatory Bowel Disease (IOIBD). Geographical Variability and Environmental Risk Factors in Inflammatory Bowel Disease. Gut 2013, 62, 630. [CrossRef]

5. Wang, K.; Wu, L.; Dou, C.; Guan, X.; Wu, H.; Liu, H. Research Advance in Intestinal Mucosal Barrier and Pathogenesis of Crohn's Disease. Gastroenterol. Res. Pract. 2016, 2016, 9686238. [CrossRef]

6. Geremia, A.; Biancheri, P.; Allan, P.; Corazza, G.R.; Sabatino, A.D. Innate and Adaptive Immunity in Inflammatory Bowel Disease. Autoimmun. Rev. 2014, 13, 3-10. [CrossRef]

7. Ahmed, I.; Roy, B.C.; Khan, S.A.; Septer, S.; Umar, S. Microbiome, Metabolome and Inflammatory Bowel Disease. Microorganisms 2016, 4, 20. [CrossRef]

8. Nishida, A.; Inoue, R.; Inatomi, O.; Bamba, S.; Naito, Y.; Andoh, A. Gut Microbiota in the Pathogenesis of Inflammatory Bowel Disease. Clin. J. Gastroenterol. 2018, 11,1-10. [CrossRef]

9. Walters, W.A.; Xu, Z.; Knight, R. Meta-analyses of Human Gut Microbes Associated with Obesity and IBD. FEBS Lett. 2014, 588, 4223-4233. [CrossRef]

10. Gevers, D.; Kugathasan, S.; Knights, D.; Kostic, A.D.; Knight, R.; Xavier, R.J. A Microbiome Foundation for the Study of Crohn's Disease. Cell Host Microbe 2017, 21, 301-304. [CrossRef]

11. Mentella, M.C.; Scaldaferri, F.; Pizzoferrato, M.; Gasbarrini, A.; Miggiano, G.A.D. Nutrition, IBD and Gut Microbiota: A Review. Nutrients 2020, 12, 944. [CrossRef] [PubMed]

12. Darfeuille-Michaud, A.; Boudeau, J.; Bulois, P.; Neut, C.; Glasser, A.-L.; Barnich, N.; Bringer, M.-A.; Swidsinski, A.; Beaugerie, L.; Colombel, J.-F. High Prevalence of Adherent-Invasive Escherichia coli Associated with Ileal Mucosa in Crohn's Disease. Gastroenterology 2004, 127, 412-421. [CrossRef] [PubMed]

13. Schippa, S.; Conte, M.P.; Borrelli, O.; Iebba, V.; Aleandri, M.; Seganti, L.; Longhi, C.; Chiarini, F.; Osborn, J.; Cucchiara, S. Dominant Genotypes in Mucosa-Associated Escherichia coli Strains from Pediatric Patients with Inflammatory Bowel Disease. Inflamm. Bowel Dis. 2009, 15, 661-672. [CrossRef] [PubMed]

14. Thomazini, C.M.; Samegima, D.A.G.; Rodrigues, M.A.M.; Victoria, C.R.; Rodrigues, J. High Prevalence of Aggregative Adherent Escherichia coli Strains in the Mucosa-Associated Microbiota of Patients with Inflammatory Bowel Diseases. Int. J. Med. Microbiol. 2011, 301, 475-479. [CrossRef] [PubMed]

15. Glasser, A.-L.; Boudeau, J.; Barnich, N.; Perruchot, M.-H.; Colombel, J.-F.; Darfeuille-Michaud, A. Adherent Invasive Escherichia coli Strains from Patients with Crohn's Disease Survive and Replicate within Macrophages without Inducing Host Cell Death. Infect. Immun. 2001, 69, 5529-5537. [CrossRef] [PubMed]

16. Eaves-Pyles, T.; Allen, C.A.; Taormina, J.; Swidsinski, A.; Tutt, C.B.; Jezek, G.E.; Islas-Islas, M.; Torres, A.G. Escherichia coli Isolated from a Crohn's Disease Patient Adheres, Invades, and Induces Inflammatory Responses in Polarized Intestinal Epithelial Cells. Int. J. Med. Microbiol. 2008, 298, 397-409. [CrossRef] [PubMed]

17. Rolhion, N.; Darfeuille-Michaud, A. Adherent-Invasive Escherichia coli in Inflammatory Bowel Disease. Inflamm. Bowel Dis. 2007, 13, 1277-1283. [CrossRef]

18. Boudeau, J.; Barnich, N.; Darfeuille-Michaud, A. Type 1 Pili-mediated Adherence of Escherichia coli Strain LF82 Isolated from Crohn's Disease Is Involved in Bacterial Invasion of Intestinal Epithelial Cells. Mol. Microbiol. 2001, 39, 1272-1284. [CrossRef]

19. Barnich, N.; Boudeau, J.; Claret, L.; Darfeuille-Michaud, A. Regulatory and Functional Co-operation of Flagella and Type 1 Pili in Adhesive and Invasive Abilities of AIEC Strain LF82 Isolated from a Patient with Crohn's Disease. Mol. Microbiol. 2003, 48, 781-794. [CrossRef]

20. Cieza, R.J.; Hu, J.; Ross, B.N.; Sbrana, E.; Torres, A.G. The IbeA Invasin of Adherent-Invasive Escherichia coli Mediates Interaction with Intestinal Epithelia and Macrophages. Infect. Immun. 2015, 83, 1904-1918. [CrossRef]

21. Chassaing, B.; Darfeuille-Michaud, A. The $\sigma^{\mathrm{E}}$ Pathway Is Involved in Biofilm Formation by Crohn's Disease-Associated AdherentInvasive Escherichia coli. J. Bacteriol. 2013, 195, 76-84. [CrossRef] [PubMed]

22. Fanelli, G.; Pasqua, M.; Colonna, B.; Prosseda, G.; Grossi, M. Expression Profile of Multidrug Resistance Efflux Pumps During Intracellular Life of Adherent-Invasive Escherichia coli Strain LF82. Front. Microbiol. 2020, 11, 1935. [CrossRef] [PubMed]

23. D’Haens, G.R.; Vermeire, S.; Assche, G.V.; Noman, M.; Aerden, I.; Olmen, G.V.; Rutgeerts, P. Therapy of Metronidazole With Azathioprine to Prevent Postoperative Recurrence of Crohn's Disease: A Controlled Randomized Trial. Gastroenterology 2008, 135, 1123-1129. [CrossRef] [PubMed]

24. Shin, S.J.; Collins, M.T. Thiopurine Drugs Azathioprine and 6-Mercaptopurine Inhibit Mycobacterium paratuberculosis Growth In Vitro. Antimicrob. Agents Chemother. 2008, 52, 418-426. [CrossRef]

25. Migliore, F.; Macchi, R.; Landini, P.; Paroni, M. Phagocytosis and Epithelial Cell Invasion by Crohn's Disease-Associated AdherentInvasive Escherichia coli Are Inhibited by the Anti-Inflammatory Drug 6-Mercaptopurine. Front. Microbiol. 2018, 9, 964. [CrossRef]

26. Nitzan, O.; Elias, M.; Peretz, A.; Saliba, W. Role of Antibiotics for Treatment of Inflammatory Bowel Disease. World J. Gastroenterol. 2016, 22, 1078. [CrossRef]

27. Ganji-Arjenaki, M.; Rafieian-Kopaei, M. Probiotics Are a Good Choice in Remission of Inflammatory Bowel Diseases: A Meta Analysis and Systematic Review. J. Cell Physiol. 2018, 233, 2091-2103. [CrossRef]

28. Ghouri, Y.A.; Richards, D.M.; Rahimi, E.F.; Krill, J.T.; Jelinek, K.A.; DuPont, A.W. Systematic Review of Randomized Controlled Trials of Probiotics, Prebiotics, and Synbiotics in Inflammatory Bowel Disease. Clin. Exp. Gastroenterol. 2014, 7, 473-487. [CrossRef] 
29. Butterworth, A.D.; Thomas, A.G.; Akobeng, A.K. Probiotics for Induction of Remission in Crohn's Disease. Cochrane Database Syst. Rev. 2008, CD006634. [CrossRef]

30. Leccese, G.; Bibi, A.; Mazza, S.; Facciotti, F.; Caprioli, F.; Landini, P.; Paroni, M. Probiotic Lactobacillus and Bifidobacterium Strains Counteract Adherent-Invasive Escherichia coli (AIEC) Virulence and Hamper IL-23/Th17 Axis in Ulcerative Colitis, but Not in Crohn's Disease. Cells 2020, 9, 1824. [CrossRef]

31. Durand, J.M.B.; Björk, G.R. Metabolic Control through Ornithine and Uracil of Epithelial Cell Invasion by Shigella flexneri. Microbiology 2009, 155, 2498-2508. [CrossRef]

32. Ueda, A.; Attila, C.; Whiteley, M.; Wood, T.K. Uracil Influences Quorum Sensing and Biofilm Formation in Pseudomonas aeruginosa and Fluorouracil Is an Antagonist. Microb. Biotechnol. 2009, 2, 62-74. [CrossRef] [PubMed]

33. Boudeau, J.; Glasser, A.-L.; Masseret, E.; Joly, B.; Darfeuille-Michaud, A. Invasive Ability of an Escherichia coli Strain Isolated from the Ileal Mucosa of a Patient with Crohn's Disease. Infect. Immun. 1999, 67, 4499-4509. [CrossRef] [PubMed]

34. Bringer, M.-A.; Rolhion, N.; Glasser, A.-L.; Darfeuille-Michaud, A. The Oxidoreductase DsbA Plays a Key Role in the Ability of the Crohn's Disease-Associated Adherent-Invasive Escherichia coli Strain LF82 To Resist Macrophage Killing. J. Bacteriol. 2007, 189, 4860-4871. [CrossRef] [PubMed]

35. Thompson, A.P.; O’Neill, I.; Smith, E.J.; Catchpole, J.; Fagan, A.; Burgess, K.E.V.; Carmody, R.J.; Clarke, D.J. Glycolysis and Pyrimidine Biosynthesis Are Required for Replication of Adherent-Invasive Escherichia coli in Macrophages. Microbiology 2016, 162, 954-965. [CrossRef] [PubMed]

36. Garavaglia, M.; Rossi, E.; Landini, P. The Pyrimidine Nucleotide Biosynthetic Pathway Modulates Production of Biofilm Determinants in Escherichia coli. PLoS ONE 2012, 7, e31252. [CrossRef]

37. Genevaux, P.; Bauda, P.; DuBow, M.S.; Oudega, B. Identification of Tn 10 Insertions in the RfaG, RfaP, and GalU Genes Involved in Lipopolysaccharide Core Biosynthesis That Affect Escherichia coli Adhesion. Arch. Microbiol. 1999, 172, 1-8. [CrossRef]

38. Møller, A.K.; Leatham, M.P.; Conway, T.; Nuijten, P.J.M.; de Haan, L.A.; Krogfelt, K.A.; Cohen, P.S. An Escherichia coli MG1655 Lipopolysaccharide Deep-Rough Core Mutant Grows and Survives in Mouse Cecal Mucus but Fails To Colonize the Mouse Large Intestine. Infect. Immun. 2003, 71, 2142-2152. [CrossRef]

39. Dartigalongue, C.; Missiakas, D.; Raina, S. Characterization of the Escherichia coli $\sigma^{\mathrm{E}}$ Regulon. J. Biol. Chem. 2001, 276, 20866-20875. [CrossRef]

40. Tam, C.; Missiakas, D. Changes in Lipopolysaccharide Structure Induce the $\sigma^{\mathrm{E}}$-dependent Response of Escherichia coli. Mol. Microbiol. 2005, 55, 1403-1412. [CrossRef]

41. Römling, U.; Rohde, M.; Olsén, A.; Normark, S.; Reinköster, J. AgfD, the Checkpoint of Multicellular and Aggregative Behaviour in Salmonella typhimurium Regulates at Least Two Independent Pathways. Mol. Microbiol. 2000, 36, 10-23. [CrossRef] [PubMed]

42. Zogaj, X.; Nimtz, M.; Rohde, M.; Bokranz, W.; Römling, U. The Multicellular Morphotypes of Salmonella typhimurium and Escherichia coli Produce Cellulose as the Second Component of the Extracellular Matrix. Mol. Microbiol. 2001, 39, 1452-1463. [CrossRef] [PubMed]

43. Herrlinger, K.R.; Diculescu, M.; Fellermann, K.; Hartmann, H.; Howaldt, S.; Nikolov, R.; Petrov, A.; Reindl, W.; Otte, J.M.; Stoynov, S.; et al. Efficacy, Safety and Tolerability of Vidofludimus in Patients with Inflammatory Bowel Disease: The ENTRANCE Study. J. Crohn's Colitis 2013, 7, 636-643. [CrossRef]

44. Ellis, M.J.; Tsai, C.N.; Johnson, J.W.; French, S.; Elhenawy, W.; Porwollik, S.; Andrews-Polymenis, H.; McClelland, M.; Magolan, J.; Coombes, B.K.; et al. A Macrophage-Based Screen Identifies Antibacterial Compounds Selective for Intracellular Salmonella typhimurium. Nat. Commun. 2019, 10, 197. [CrossRef]

45. Bange, F.C.; Brown, A.M.; Jacobs, W.R. Leucine Auxotrophy Restricts Growth of Mycobacterium bovis BCG in Macrophages. Infect. Immun. 1996, 64, 1794-1799. [CrossRef]

46. Pilatz, S.; Breitbach, K.; Hein, N.; Fehlhaber, B.; Schulze, J.; Brenneke, B.; Eberl, L.; Steinmetz, I. Identification of Burkholderia pseudomallei Genes Required for the Intracellular Life Cycle and In Vivo Virulence. Infect. Immun. 2006, 74, 3576-3586. [CrossRef]

47. Smith, D.A.; Parish, T.; Stoker, N.G.; Bancroft, G.J. Characterization of Auxotrophic Mutants of Mycobacterium tuberculosis and Their Potential as Vaccine Candidates. Infect. Immun. 2001, 69, 1142-1150. [CrossRef] [PubMed]

48. Beaumont, H.J.E.; Gallie, J.; Kost, C.; Ferguson, G.C.; Rainey, P.B. Experimental Evolution of Bet Hedging. Nature 2009, 462, 90. [CrossRef]

49. Tükel, Ç.; Nishimori, J.H.; Wilson, R.P.; Winter, M.G.; Keestra, A.M.; Putten, J.P.M.V.; Bäumler, A.J. Toll-like Receptors 1 and 2 Cooperatively Mediate Immune Responses to Curli, a Common Amyloid from Enterobacterial Biofilms. Cell Microbiol. 2010, 12, 1495-1505. [CrossRef]

50. Rapsinski, G.J.; Wynosky-Dolfi, M.A.; Oppong, G.O.; Tursi, S.A.; Wilson, R.P.; Brodsky, I.E.; Tükel, Ç. Toll-like Receptor 2 and NLRP3 Cooperate to Recognize a Functional Bacterial Amyloid, Curli. Infect. Immun. 2014, 83, 693-701. [CrossRef]

51. Lauritsen, I.; Frendorf, P.O.; Capucci, S.; Heyde, S.A.H.; Blomquist, S.D.; Wendel, S.; Fischer, E.C.; Sekowska, A.; Danchin, A.; Nørholm, M.H.H. Temporal Evolution of Master Regulator Crp Identifies Pyrimidines as Catabolite Modulator Factors. Nat. Commun. 2021, 12, 5880. [CrossRef] [PubMed]

52. Rossi, E.; Motta, S.; Aliverti, A.; Cossu, F.; Gourlay, L.; Mauri, P.; Landini, P. Cellulose Production Is Coupled to Sensing of the Pyrimidine Biosynthetic Pathway via c-di-GMP Production by the DgcQ Protein of Escherichia coli. Environ. Microbiol. 2017, 19, 4551-4563. [CrossRef] 
53. Muehler, A.; Kohlhof, H.; Groeppel, M.; Vitt, D. Safety, Tolerability and Pharmacokinetics of Vidofludimus Calcium (IMU-838) After Single and Multiple Ascending Oral Doses in Healthy Male Subjects. Eur. J. Drug Metab. Pharmacokinet. 2020, 45, 557-573. [CrossRef] [PubMed]

54. Antoniani, D.; Rossi, E.; Rinaldo, S.; Bocci, P.; Lolicato, M.; Paiardini, A.; Raffaelli, N.; Cutruzzolà, F.; Landini, P. The Immunosuppressive Drug Azathioprine Inhibits Biosynthesis of the Bacterial Signal Molecule Cyclic-Di-GMP by Interfering with Intracellular Nucleotide Pool Availability. Appl. Microbiol. Biot. 2013, 97, 7325-7336. [CrossRef] [PubMed]

55. Leclercq, R.; Courvalin, P. Intrinsic and Unusual Resistance to Macrolide, Lincosamide, and Streptogramin Antibiotics in Bacteria. Antimicrob. Agents Chemother. 1991, 35, 1273-1276. [CrossRef] [PubMed]

56. Nørager, S.; Jensen, K.F.; Björnberg, O.; Larsen, S. E. Coli Dihydroorotate Dehydrogenase Reveals Structural and Functional Distinctions between Different Classes of Dihydroorotate Dehydrogenases. Structure 2002, 10, 1211-1223. [CrossRef]

57. Marcinkeviciene, J.; Rogers, M.J.; Kopcho, L.; Jiang, W.; Wang, K.; Murphy, D.J.; Lippy, J.; Link, S.; Chung, T.D.Y.; Hobbs, F.; et al. Selective Inhibition of Bacterial Dihydroorotate Dehydrogenases by Thiadiazolidinediones. Biochem. Pharmacol. 2000, 60, 339-342. [CrossRef] 